\title{
Racionamiento vía listas de espera: medidas de mejora y posibles implicaciones
}

\author{
Rationing through waiting lists: measuring \\ improvement and possible implications
}

\footnotetext{
${ }^{1}$ Facultad de Ciencias Económicas y Empresariales, Universidad de Vigo, Vigo, España.

2 Facultad de Ciencias Económicas y Empresariales, Universidad de Barcelona, Barcelona, España.

Correspondencia E. Rodríguez

Departamento de Economía Aplicada, Facultad de Ciencias Económicas y Empresariales, Universidad de Vigo.

Campus As Lagoas-Marcosende, 36310 Vigo, España.

emiguez@uvigo.es
}

\section{Abstract}

This paper analyzes the main policy initiatives for improving waiting lists in health care. The authors begin by describing strategies to reduce either waiting time or length of the list. They distinguish between demand-side and supplyside strategies. They proceed to discuss policies for improving the "quality" of waiting time. For each policy, they present both the expected effect and the indirect effects that can reduce its effectiveness for improving waiting list conditions.

Waiting Lists; Health Policy; Health Management
Eva Rodríguez 1

Begoña Álvarez ${ }^{1}$

Pilar Abad 2

\section{Introducción}

Los países que ofrecen los cuidados sanitarios a través de un sistema nacional de salud, con nulos o reducidos copagos, suelen caracterizarse por la existencia de listas de espera en la provisión de determinados servicios, principalmente, visitas al especialista, pruebas diagnósticas e intervenciones de cirugía electiva. La existencia de listas de espera responde al hecho de que la recepción de un determinado servicio no lleva implícito el pago de ningún precio, lo que suele provocar una demanda de servicios superior a la oferta disponible. Las listas de espera no serían un problema, es más serían deseables para lograr una gestión eficiente de los recursos 1, si el tiempo de espera para recibir la atención requerida fuese "razonable". Sin embargo, en la actualidad las listas de espera constituyen uno de los problemas más importantes que sufren los sistemas nacionales de salud, debido al excesivo tiempo de espera que conllevan determinados servicios. Esta espera puede ser especialmente traumática si implica un deterioro irrecuperable de la salud o, en situaciones extremas, la muerte del paciente.

En este artículo se analizan las principales estrategias que se han planteado en la gestión sanitaria para tratar de mejorar la situación de las listas de espera, tanto desde el punto de vista de la eficiencia como de la equidad. Para facilitar su presentación, las hemos organizado atendiendo a su finalidad. En primer lugar, se describen las 
estrategias que contribuyen, directa o indirectamente, a mejorar los indicadores tradicionales (tiempo medio de espera y tamaño de la lista). Dentro de este grupo se analizan tanto las medidas destinadas a incrementar la oferta, principalmente aumentos presupuestarios y mejoras en la gestión, como aquéllas orientadas a controlar la demanda. En segundo lugar, se muestran las estrategias cuyo objetivo principal no es mejorar los indicadores tradicionales, sino mejorar la calidad de la espera.

\section{¿Pueden los incrementos de recursos mejorar los indicadores de listas de espera?}

Una de las estrategias más obvias para mejorar los indicadores de las listas de espera es la inyección de recursos adicionales al sistema. Esto se puede realizar a través de un incremento de los recursos del sistema público de salud o a través de la concertación con el sector privado.

En general, las comparaciones internacionales sugieren la existencia de una relación negativa entre recursos disponibles y el tamaño de las listas de espera 2 . Sin embargo, la mayoría de los estudios no considera las particularidades de cada sistema sanitario, por ejemplo, no tienen en cuenta que los países pueden tener estructuras de copago diferentes y, por tanto, demandas de servicios diferentes. De hecho, cuando se analiza el efecto de estas medidas dentro de un mismo país, los incrementos de recursos no parecen tener efectos muy positivos sobre la dinámica de las listas de espera ${ }^{3}$. Además, estos efectos varían según se trate de un incremento de recursos coyuntural o permanente. Los incrementos permanentes, a pesar de conllevar un incremento también permanente de actividad, provocan a medio plazo un aumento del número de pacientes en espera y una reducción 4 o un estancamiento 5 en el tiempo de espera. Las medidas coyunturales como, por ejemplo, los planes de choque y los fondos finalistas destinados a garantizar tiempos máximos de espera, aunque provocan a corto plazo una reducción tanto en el número de pacientes en espera como en el tiempo de espera, su efecto desaparece en cuanto se dejan de inyectar recursos adicionales 6,7. En algunos países, como Canadá 8 y Reino Unido 9 , estos efectos "perversos" han provocado un incremento, no sólo en el tamaño de las listas, sino también en los tiempos de espera meses después de la inyección de recursos adicionales.

La escasa eficacia de este tipo de medidas viene explicada, al menos en parte, por el sistema de incentivos que se desencadenan. La au- sencia de un sistema que premie (penalice) la reducción (aumento) de las listas de espera hace que los incrementos de la oferta puedan provocar incrementos de la demanda, por ejemplo, un incremento en el número de indicaciones. Tal situación puede verse agudizada si, además, los médicos y/o los hospitales tienen incentivos económicos ligados a este incremento de demanda, por ejemplo, pago por actividad, o inyección adicional de recursos ante listas de espera elevadas 10 . Por tanto, para evitar estos efectos no deseados se debe complementar la inyección de recursos con medidas adicionales: condicionar la inyección de recursos a reducciones en el tiempo de espera; intensificar el control de la indicación de las intervenciones 11, etc.

\section{Mejorar la gestión: ¿una mejor solución?}

El incremento de recursos sin ninguna medida adicional no tiene por qué implicar una mayor productividad de los servicios. Sin embargo, existen otro tipo de estrategias que basan el incremento de oferta en un uso más eficiente de los recursos ya disponibles. Vamos a revisar las más significativas.

Un primer tipo de medidas serían los "sistemas de pago". Se trata de un instrumento muy relevante para la eficiencia de los sistemas de salud, porque las decisiones sobre gasto y nivel de producción en última instancia dependen de los profesionales sanitarios; y ocurre que las fórmulas de pago, y los incentivos que éstas generan, son capaces de influir sobre ellos. Cuando el objetivo prioritario es actuar sobre las listas de espera, se suele recomendar recurrir a fórmulas de pago que estimulen la actividad. Así, a nivel hospitalario, el paso de una financiación mediante presupuestos cerrados a una financiación por proceso o por ingreso puede estimular la actividad ${ }^{12}$. En cuanto a los médicos especialistas, un cambio en la remuneración que se aleje del pago por salario no ajustado por actividad, puede tener un efecto directo sobre las listas de espera de atención especializada y pruebas diagnósticas 13 .

Sin embargo, los sistemas de pago por actividad pueden tener efectos compensatorios en el largo plazo. Por una parte, la demanda puede aumentar como resultado de la reducción del tiempo de espera. Por otra parte, existe un mayor riesgo de reingresos (segundas consultas), derivado de la reducción en la duración media de la estancia (tiempo de atención al paciente) que suele conllevar el pago por proceso. Finalmente, suele observarse un incremento de las listas de espera en aquellos procesos que no son remunerados mediante este tipo de fórmulas, frente a 
aquéllos que sí lo son. Una forma de paliar estos incentivos perversos sería combinar esta fórmula de pago con protocolos que garanticen una adecuada indicación de los tratamientos, así como la realización de auditorías de la actividad hospitalaria.

Otro aspecto importante que puede influir considerablemente en la lista de espera es el conflicto de intereses generado en sistemas sanitarios que permiten la compatibilidad de la práctica pública (básicamente remunerada mediante salario) y privada (generalmente remunerada por actividad). Los especialistas que mantienen una práctica dual (pública y privada) pueden sentirse incentivados a mantener una lista de espera larga en los centros públicos con la finalidad de aumentar la demanda de su práctica privada ${ }^{14}$. En algunos países, existe evidencia de que los cirujanos que compatibilizan la práctica pública y privada tienen tiempos de espera en el sector público considerablemente más prolongados que sus colegas que sólo operan dentro del sistema público 15 .

Un segundo grupo de medidas son las que inciden en una mayor "coordinación entre diferentes niveles asistenciales", generalmente entre atención primaria y especializada. Estas medidas buscan principalmente reducir la presión sobre los hospitales (tanto sobre sus consultas externas como sobre sus ingresos). De especial interés son las medidas que tratan de incrementar la cirugía mayor ambulatoria y las que tratan de incrementar la capacidad resolutoria de atención primaria. La cirugía mayor ambulatoria reduce el coste de la intervención vía reducciones en el tiempo de estancia y, por tanto, conlleva una mejora de la eficiencia (se incrementa la actividad con la misma dotación de recursos). A esta mejora hay que añadir una mayor satisfacción de los pacientes. No obstante, los efectos de la coordinación entre niveles asistenciales sobre los indicadores de listas de espera no están claros. Así, mientras se consideran como una de las causas de las bajas listas de espera en Alemania, en otras regiones se ha observado que, paralelamente a la extensión de la cirugía mayor ambulatoria, aumenta el número de indicaciones. En este caso, el incremento de la demanda se produce porque las intervenciones ambulatorias conllevan un menor riesgo, lo cual reduce el rechazo por parte de los pacientes, y porque se trata de intervenciones que pueden ser indicadas en pacientes a los que se les desaconsejan tratamientos más agresivos.

Dentro de este mismo grupo de medidas estarían aquéllas que tratan de aumentar la capacidad resolutoria de la atención primaria, con el objetivo de disminuir la presión sobre la atención especializada. Se trata de proporcionar a los servicios de atención primaria un mayor protagonismo en el diagnóstico y seguimiento de algunas patologías, permitiendo una mayor dedicación del especialista a la actividad quirúrgica propiamente dicha 16 . Estas medidas no tienen por qué incidir en una menor satisfacción del paciente $17 \mathrm{y}$, sin embargo, pueden reducir las listas de espera.

Finalmente, debemos citar las medidas que tratan de mejorar la eficiencia a través de una mejora en la "gestión de los recursos físicos disponibles". El objetivo que persiguen estas medidas es mejorar el volumen de la actividad asistencial sin que apenas se altere la calidad de la misma. Un ejemplo sería la utilización de técnicas basadas en los modelos de colas, la simulación, la optimización matemática o el análisis multicriterio para incrementar la eficiencia en la asignación de camas o quirófanos. Estas técnicas tratan de identificar ineficiencias, como cuellos de botella o infrautilización de los recursos, mostrando posibles reasignaciones de recursos que generan asignaciones de gasto más eficientes 18,19. Paralelamente, estarían las medidas que buscan mejorar la gestión de la propia lista de espera como las depuraciones administrativas, las llamadas previas a la cita y las auditorías de las listas (controlan si la provisión del servicio sigue estando indicada). Estas estrategias de gestión no sólo ayudan a disponer de información más veraz sobre los pacientes que realmente están esperando, sino que pueden contribuir a la reducción real de las listas dado que evitan pérdidas innecesarias de actividad 20

\section{Estrategias basadas en el control de la demanda}

Hemos visto que los incentivos a la actividad suelen conllevar modificaciones en la demanda que anulan los efectos favorables sobre las listas de espera. Por ese motivo, las estrategias de oferta deben ser complementadas con medidas destinadas al "control de las indicaciones médicas". La evidencia empírica muestra que los criterios que guían las decisiones de indicación de tratamientos o intervenciones por parte de los profesionales sanitarios no suelen ser homogéneos entre diferentes áreas geográficas u hospitales, lo cual provoca diferencias significativas y no aleatorias en la práctica médica. Cuando las variaciones en las tasas estandarizadas de un procedimiento son sistemáticas, reciben el nombre de "variabilidad de la práctica médica" 21,22. Si esta variabilidad no se corresponde con diferencias en los patrones de morbilidad, se convierte 
en un problema, ya que pone de manifiesto la existencia de indicaciones inapropiadas de tratamientos e intervenciones 23 . Por tanto, las medidas destinadas a orientar a los profesionales sanitarios y a establecer pautas sobre indicación de tratamientos (guías de práctica clínica, protocolos de indicación, programas de segunda opinión, etc.) pueden traducirse no sólo en una reducción de las listas de espera, sino también en mejoras en la salud de los pacientes.

Otra de las alternativas para reducir la demanda sobre los servicios sanitarios públicos consiste en "incentivar la contratación de seguros privados" complementarios, por ejemplo, mediante desgravaciones fiscales. Este tipo de actuación no busca reducir la demanda global de atención médica, sino desplazar demanda hacia el ámbito privado con la finalidad de reducir las listas de espera en la atención pública. Evidentemente, los efectos sobre las listas de espera dependerán del grado de sustitución entre la atención sanitaria pública y la privada. Por ejemplo, si la atención privada fuese percibida por los ciudadanos como de menor calidad que la pública en las intervenciones graves, los incentivos a la contratación de seguros privados podrían acabar subvencionando intervenciones menores o, simplemente, aquéllas excluidas del catálogo de prestaciones sanitarias públicas. En este caso, el efecto sobre las listas de espera sería de escasa relevancia. Por otra parte, incluso si el grado de sustitución entre ambos tipos de asistencia fuese elevado, esta medida podría tener un efecto escaso a medio y largo plazo sobre las listas de espera, dado que la reducción en el tiempo de espera de la atención pública podría desincentivar la compra de un seguro privado. Además, si los médicos desarrollan su actividad en el ámbito público y privado pueden tener incentivos a mantener largas listas de espera para incentivar la compra de seguros privados.

\section{Estrategias para mejorar el output: priorización de pacientes}

Generalmente, a la hora de hacer un diagnóstico sobre la situación de las listas de espera, se suele poner un excesivo énfasis en los indicadores tradicionales. Sin embargo, también se debería tener en cuenta la situación de necesidad de los pacientes que esperan, puesto que este aspecto incide en el nivel de satisfacción con el sistema. De esta forma, paralelamente a las medidas que tratan de reducir los tiempos de espera, se debería incidir en aquellas medidas que tratan de mejorar la calidad de los que están esperando, medida ésta como salud y/o nivel bienestar.
Los "sistemas de priorización" de pacientes en lista de espera irían en esta dirección ${ }^{24}$. La ordenación de los pacientes que esperan una determinada intervención en función de su necesidad clínica no tiene por qué implicar una reducción en el tiempo de espera, pero sí garantiza que las intervenciones realizadas en primer lugar sean las que proporcionen una mayor ganancia total, entendida ésta como nivel de satisfacción, output sanitario, etc. Por tanto, los mecanismos de priorización contribuyen a una mayor equidad en el acceso y a una mejora en la situación de los pacientes que están esperando. Estos efectos podrían ser incluso mayores si la priorización se realizase sobre una única lista que incluyese a todos los pacientes de un área concreta y para una especialidad en particular 25.

Por otro lado, los sistemas de priorización contribuyen a aumentar la racionalidad a la hora de establecer tiempos máximos de espera garantizados. Si se fijan tiempos máximos de espera diferentes según el nivel de prioridad, se reduce la probabilidad de que pacientes en situación menos urgente, que alcanzan el tiempo máximo establecido, sean atendidos antes que otros enfermos con mayor gravedad clínica pero con un menor tiempo de espera.

En los sistemas nacionales de salud es habitual que se establezcan criterios de priorización generales (por ejemplo, urgente y no urgente), pero es poco frecuente que se prioricen los pacientes no urgentes en base a su necesidad clínica o social. De hecho, el único criterio que utilizan la mayoría de sistemas sanitarios para priorizar a estos pacientes es el tiempo de espera. Sin embargo, existen experiencias internacionales que tratan de incorporar mecanismos de priorización basados en la necesidad del paciente. En algunos países se utilizan criterios exclusivamente clínicos, generalmente en situaciones de urgencia 26,27 , mientras que otros combinan criterios clínicos y sociales a la hora de determinar la necesidad de un paciente 28,29. Evidentemente, estos sistemas de priorización, conducen a una ordenación de los pacientes diferente de la que resultaría en función de un criterio basado exclusivamente en el tiempo de espera 29. Algunos de estos sistemas de priorización pueden obtenerse a partir de las preferencias de la sociedad, lo cual incrementa su legitimidad y, posiblemente, su nivel de aceptación 30 .

\section{Conclusiones}

De la exposición anterior se deduce que no existe una medida natural que solucione el problema de las listas de espera, debido a los incentivos 
que cada una de ellas puede desencadenar. Sin embargo, sí podemos hacer algunas reflexiones generales que pueden ser importantes a la hora de poner en marcha políticas para mejorar las listas de espera.

En primer lugar, una de las conclusiones que podemos extraer de la presentación anterior es que cualquier incremento de los recursos sanitarios disponibles suele estimular a medio plazo la demanda, eliminando la mejora inicial de los indicadores. ¿ Cuestiona este resultado la eficacia de las medidas basadas en los incrementos de recursos? No, si ese incremento de la demanda responde a una demanda latente que estaba siendo ignorada. Por el contrario, serían ineficaces si incrementan la actividad con escasa contribución sobre la salud. Por ese motivo es necesario combinar los incrementos de recursos con estrategias de control de demanda, como por ejemplo los protocolos de indicación médica, que eviten la incorporación a la lista de nuevos pacientes con una indicación de tratamiento dudosa.

\section{Resumen}

En este trabajo se discuten las principales estrategias que se han planteado en la gestión sanitaria para mejorar la situación de las listas de espera. En primer lugar, se describen aquellas medidas que tratan de mejorar los indicadores tradicionales de las listas de espera (tiempo de espera y tamaño de la lista), distinguiendo entre aquéllas que tratan de incidir en el incremento de la oferta y las que tratan de controlar la demanda. En segundo lugar, se analizan aquellas medidas que no implican necesariamente una mejora en los indicadores tradicionales, pero sí consiguen mejoras significativas en la calidad de la espera. Para cada una de las estrategias se muestran tanto sus efectos esperados como otros efectos colaterales que alertan sobre su eficacia a la hora de mejorar la situación de las listas de espera.

Listas de Espera; Política de Salud; Gestión en Salud
En segundo lugar, resulta necesario avanzar en las estrategias destinadas a la priorización de aquellos pacientes que están esperando un determinado servicio. Una priorización adecuada de pacientes no incide directamente sobre los indicadores de lista de espera, pero sí actúa sobre la eficiencia y la equidad de su gestión. Sobre la eficiencia porque las intervenciones realizadas en primer lugar son las que proporcionan una mayor ganancia total, entendida ésta como nivel de satisfacción, output sanitario, etc. Si el criterio de ordenación de los pacientes en lista de espera alcanza este objetivo, contribuirá a un mayor bienestar social. Respecto a la equidad, la priorización de pacientes en base a la necesidad garantiza que pacientes con un mismo nivel de necesidad tengan el mismo acceso a los tratamientos médicos.

Evidentemente, la efectividad de estas estrategias también dependerá del desarrollo de medidas complementarias orientadas a la racionalización en la gestión de los recursos físicos y humanos disponibles en cada centro.

\section{Colaboradores}

Los tres autores participaron en todas las etapas de producción del artículo.

\section{Agradecimientos}

Este trabajo forma parte de un projecto de investigación financiado por la Fundación Pedro Barrié de la Maza. 


\section{Referencias}

1. Cullis J, Jones P, Propper C. Waiting lists and medical treatment: analysis and policies. In: $\mathrm{Cu}-$ lyer AJ, Newhouse JP, editors. Handbook of health economics. Amsterdam: North Holland; 2000. p. 1201-49.

2. Siciliani L, Hurst J. Explaining waiting times variations for elective surgery across OECD countries. Paris: Organisation for Economic Co-operation and Development; 2003. (OECD Economic Studies, 38).

3. Sanmartin C. Establishing acceptable waiting times for medical services: a review of the evidence and proposed methods (Appendix G). In: Western Canada Waiting List Project, editor. From chaos to order: making sense of waiting lists in Canada. Final report. Calgary: Western Canada Waiting List Project; 2001. p. 373-408.

4. Newton JN, Henderson J, Goldacre MJ. Waiting list dynamics and the impact of earmarked funding. BMJ 1995; 311:783-5.

5. Hamblin R, Harrison A, Boyle S. Waiting lists. The wrong target. Health Serv J 1998; 108:28-31.

6. Mobb GE, Pugh F, Peeling B. How long is your waiting list? Experience of a urological waiting list initiative. J R Soc Med 1994; 87:140-2.

7. Hanning M. Maximum waiting-time guarantee - an attempt to reduce waiting lists in Sweden. Health Policy 1996; 36:17-35.

8. DeCoster C, Carrière K, Peterson S, Wald R, MacWilliam L. Surgical waiting times in Manitoba. Winnipeg: Manitoba Centre for Health Policy and Evaluation; 1998.

9. Appleby J. Waiting times go down, but waiting lists up. BMJ 1993; 306:479.

10. Duckett S, Street A. Are waiting lists inevitable? Health Policy 1996; 36:1-15.

11. Nordberg M, Keskimaki I, Hemminki E. Is there a relation between waiting-list length and surgery rate? Int J Health Plann Manage 1994; 9:259-65.

12. Biørn E, Hagen T, Iversen T, Magnussen J. The effect of activity-based financing on hospital efficiency: a panel data analysis of DEA efficiency scores 1992-2000. Oslo: Health Economics Research Programme, University of Oslo; 2002. (Working Paper, 8).

13. Siciliani L, Hurst J. Tackling excessive waiting times for elective surgery: a comparative analysis of policies in 12 OECD countries. Health Policy 2005; 72:201-15.

14. Bloom BS, Fendrick AM. Waiting for care: queuing and resource allocation. Med Care 1987; 25:131-9.

15. DeCoster C, Carriere KC, Peterson S, Walld R, MacWilliam L. Waiting times for surgical procedures. Med Care 1999; 37:187-205.
16. Freeman G, Hjortdahl P. What future for continuity of care in general practice? BMJ 1997; 314:1870-3.

17. Haggerty JL, Reid RJ, Freeman GK, Starfield BH, Adair CE, McKendry R. Continuity of care: a multidisciplinary review. BMJ 2003; 327:1219-21.

18. Cerdá E, de Pablos L, Rodríguez MV. La gestión de las listas de espera quirúrgica en España. Instituto de Estudios Fiscales, Ministerio de Economía y Hacienda; 2002. (Documentos de Trabajo, 3/02).

19. Vissers JMK, van der Bij JD, Kusters RJ. Towards decision support for waiting lists: an operations management view. Health Care Manag Sci 2001; 4:133-42.

20. Williams RL, Williams LA, Watura R. Impact of MRI on knee arthroscopy waiting list. Ann R Coll Surg Engl 1996; 78:450-2.

21. Fisher ES, Wennberg DE, Stukel TA, Gottlieb DJ, Lucs FL, Pinder EL. The implications of regional variations in Medicare spending. Part 1: the content, quality, and accessibility of care. Ann Intern Med 2003; 138:288-98.

22. Grupo VPM-IRYS. Variaciones en cirugía ortopédica y traumatología en el Sistema Nacional de Salud. Atlas de Variación de la Práctica Médica en el Sistema Nacional de Salud. 2005; 1:27-46.

23. Gertman PM, Restuccia JD. The appropriateness evaluation protocol: a technique for assessing unnecessary days of hospital care. Med Care 1981; 19:855-71.

24. Pinto JL, Castells X, Gracia X, Sánchez F. El establecimiento de prioridades en la cirugía electiva. Barcelona: Ministerio de Sanidad y Consumo/Centro de Investigación en Economía y Salud, Universidad Pompeu Fabra; 2000.

25. Lewis S, Barer ML, Sanmartin C, Sheps S, Shortt SED. Ending waiting-list mismanagement: principles and practice. CMAJ 2000; 162:1297-300.

26. Browning CJ, Thomas SA. Community values and preferences in transplantation organ allocation decisions. Soc Sci Med 2001; 52:853-61.

27. Ratcliffe J. Public preferences for the allocation of donor liver grafts for transplantation. Health Econ 2000; 9:137-48.

28. Hadorn DC, Holmes AC. The New Zealand priority criteria project. Part 1: overview. BMJ 1997; 314:131-4.

29. Lack A, Edwards RT, Boland A. Weights for waits: lessons from Salisbury. J Health Serv Res Policy 2000; 50:83-8.

30. Espallargues M, Sampietro L, Rodríguez E, Pinto JL, Comas M, Castells X, et al. Wide social participation in prioritizing patients on waiting lists for joint replacement: a conjoint analysis. Med Decis Making; en prensa.

Recibido el 05/Nov/2007

Aprobado el 10/Ene/2008 